\title{
A GIS-Based Decision Support System for Reducing Air Ambulance Response Times: A Case Study on Public Schools in Jeddah City
}

\author{
Randa Alharbi \\ Department of Geography and GIS, King Abdulaziz University, Jeddah, Saudi Arabia \\ Email: randa.alharbi@gmail.com
}

Received 8 June 2015; accepted 9 August 2015; published 12 August 2015

Copyright (C) 2015 by author and Scientific Research Publishing Inc.

This work is licensed under the Creative Commons Attribution International License (CC BY).

http://creativecommons.org/licenses/by/4.0/

(c) (i) Open Access

\begin{abstract}
In injuries reducing ambulance response time (time from injury to hospital arrival) is an important factor for saving people's lives. Helicopter emergency medical services can reduce out-ofhospital transport times because of their high speed and their ability to travel in straight paths unlike ground ambulance which are restricted to road network paths, as well as the ability to access rural or remote area injuries that are difficult to reach by ground ambulance. GIS technology aids air ambulance movement planning to reduce out-of-hospital response time based on mathematical and geographic models to support decision making which is necessary from out-ofhospital care providers. The goal of this study is to use GIS to develop an efficient DSS to outline where air ambulance can reduce response times, by using spatial analysis tools to create Euclidean distance and direction zones around receiving hospitals. The study concludes that GIS technology can be used to develop an efficient DSS to outline where air ambulance can reduce response times, by creating surfaces of Euclidean allocation, direction, and distance that can be used to improve initial response times for the civil defense air rescue and air ambulance services.
\end{abstract}

\section{Keywords}

GIS, DSS, Air Ambulance, Response Time, Jeddah

\section{Introduction}

In recent years, population growth in Jeddah City is increasing rapidly along with traffic problems in a city where car travel demand and density are very high due to the absence of public transportation.

The lack of public awareness of traffic rules causes many traffic problems such as traffic jams and travel time 
delay. However, in certain situations it is crucial to reduce travel time.

In injuries reducing ambulance response time (time from injury to hospital arrival) is an important factor for saving people's lives.

Helicopter emergency medical services can reduce out-of-hospital transport times because of their high speed and their ability to travel in straight paths unlike ground ambulance which are restricted to road network paths, as well as the ability to access rural or remote area injuries that are difficult to reach by ground ambulance.

In some cases there are delays in flight caused by flight preparation and travel time from the helicopter base to the reported location. Therefore use of air ambulance must be properly operated to gain its time advantage over ground ambulance to be recognized, as time saving is the main advantage of air ambulance.

"Geographic information systems (GIS) are computer-based systems for the integration and analysis of geographic data. GIS is a multi-layering mapping software that is able to portray multiple geographic-time information in an easy-to-read, graphical manne" [1].

GIS technology aids air ambulance movement planning to reduce out-of-hospital response time based on mathematical and geographic models to support decision making which is necessary from dispatchers.

The hypothesis of this study is that using GIS to develop an efficient DSS to outline where air ambulance can reduce response times, by using spatial analysis tools to create Euclidean distance and direction zones around receiving hospitals.

\section{Background}

Geographic information system (GIS) is a system that transforms data collected from various sources and gives a graphical and visual representation of the information. Also GIS can communicate with other programs such as spreadsheet database systems, web-browsers and is easily integrated into programming languages making it a very powerful tool to develop applications that have spatial dimensions.

According to [2], a GIS is composed of people (the users of the system), applications (the processes and programs they use to do their work), data (the information needed to support those applications), software (the core GIS software) and hardware (the physical components on which the system runs). Figure 1 illustrates GIS components:

A GIS can be a very powerful tool in emergency management. This technology has the ability to capture the data by digitizing, scanning, digital imagery, or aerial photography; to store the data; to manipulate the data; to form data queries; to analyze the data; and most importantly, to visualize the data. In other words, GIS technology brings to the user the ability to integrate, store, process, and output geographic information. This system takes a multitude of data from numerous sources and graphically displays the information [3].

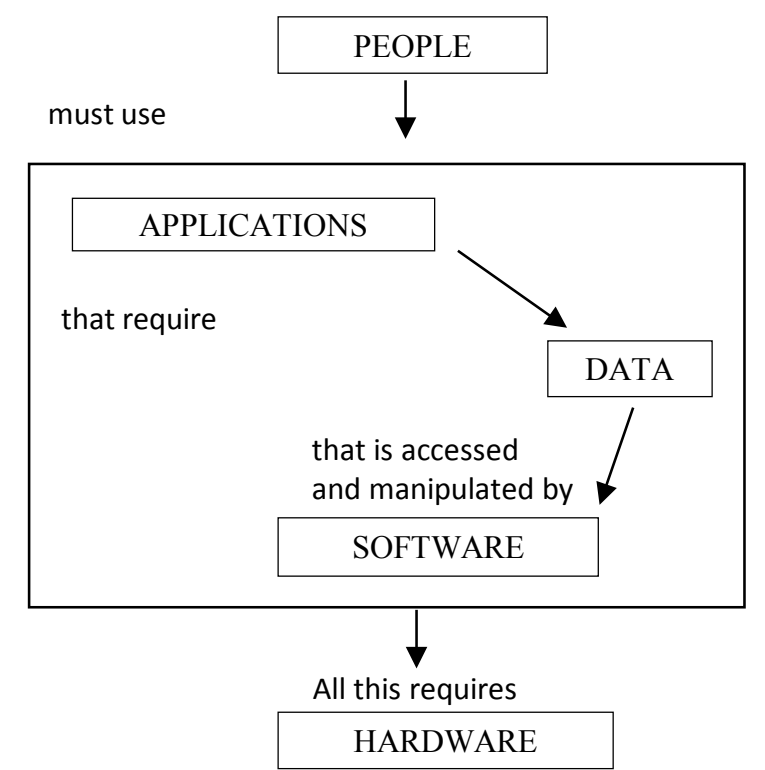

Figure 1. Components of an Enterprise GIS [2]. 
Geographic access to emergency treatment remains an important public policy concern as rural emergency medical systems respond to various pressures to centralize services. GIS has effective tools to determine what proportion of a given population is adequately served by existing or proposed service distributions [4].

Using GIS in emergency vehicles offers a valuable tool for network analysis, visualization and management. It generally offers tools to find the shortest or fastest route through a network. These capabilities of GIS for analyzing spatial networks enable it to be used as a decision support system for dispatching and routing of vehicles. The efficient management of ambulance routing to the incident sites and then to the closest hospitals is a vital aspect of the quality of health services offered to citizens. Accomplishing an effective ambulance routing minimizes its response time and thus improves the response performance [5].

GIS based protocols for location of emergency medical resources can provide supportive evidence for allocation decisions - especially when resources are limited [6].

A GIS can be used to model a given region to define where air ambulance transport will minimize out ofhospital time and result in a shorter arrival time at a hospital. In certain situations, helicopter emergency medical services (HEMS) can shorten out-of-hospital transport times because helicopters travel at higher rates of speed (i.e., 120 - $180 \mathrm{mph} 1$ ) and follow more direct routes than ground ambulances. However, in some instances it may not be advantageous to transport by HEMS because of delays due to flight preparation and distance from the helicopter base to the injury location. This is particularly true when patients can be quickly prepared for ambulance transport from the scene of injury. In general, patients benefit from helicopter transport only if there is a significant reduction in out-of-hospital transport time [7].

\section{Air Ambulance in Saudi Arabia}

The Saudi Air Ambulance service is an emergency medical helicopter service which provides air transport for the critically ill or injured. The latest and most radical initiatives in the Saudi Red Crescent's (SRC) mission 'Striving to Save Lives', the service will significantly reduce the time taken to reach critically ill and injured patients. This benefit is two-fold: emergency medical teams are able to rapidly reach and attend to the patient at the scene, and the Air Ambulance is then able to transport the stabilized patient to the hospital in the shortest time possible, maximizing the chance of full recovery (Figure 2).

Table 1 shows the type and quantity of helicopters available in the SRC Air Ambulance service.
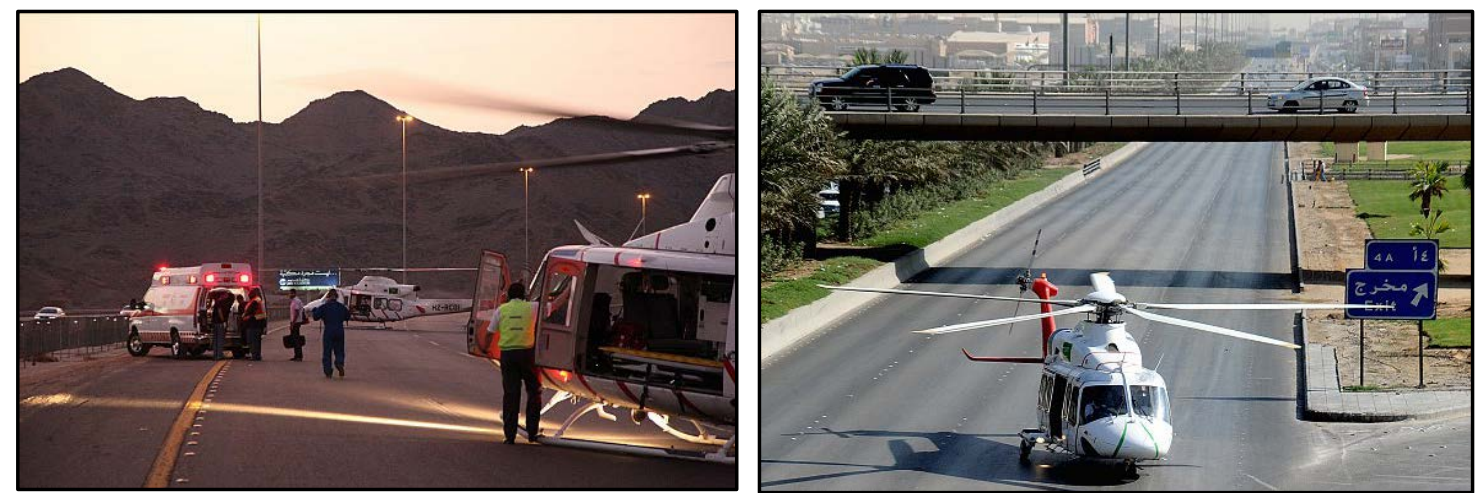

Figure 2. SRC Air Ambulance Service at the Rescue. Source: Alriyadh Electronic News http://www.alriyadh.com.

Table 1. SRC available air ambulance service.

\begin{tabular}{cc}
\hline Helicopter Model & Quantity \\
\hline Bell 412EP & 8 \\
AgustaWestland AW139 & 3 \\
Sikorsky S-76 C++ & 2 \\
Total & $\mathbf{1 3}$ \\
\hline
\end{tabular}

Source: http://en.wikipedia.org/wiki/Saudi Air Ambulance (as of 2010). 
In regards to the use of geographic information systems in the Saudi Red Crescent Authority in Jeddah city, Al-Rashdi studied its components equipment's and operations, to recognize the system's role in the Saudi Red Crescent Authority and conducted a survey upon the users of the current GIS system.

The study found that the used system is three years old at the time of the study, and had made decision making faster and more accurate than traditional methods, however many employees expressed that the system did not achieve all of the required objectives:

- The Jeddah city digital map used in the system is not updated since 2005.

- The locations are not detailed due to lack of district and street names in the geographic database.

- GPS is not activated.

- Users could not make any modification to the system without taking permission from the main center in the capital city.

- Lack of GIS and GPS expertise amongst users [8].

\section{Materials and Methods}

ArcGIS software is the leading GIS software provided by the Environmental Systems Research Institute (ESRI). It is designed to have many modules with different desktop applications known as ArcCataloge, ArcMap, Arc Globe, ArcTool box, and Model Builder. Using these applications users can perform any GIS task, from simple to advanced, including mapping, geographical analysis, data editing and completion, data management, visualization and Geoprocessing" [9].

This study is done by using ArcInfo ArcGIS desktop applications: ArcMap, Arc Catalog, and ArcToolbox.

Arc Map application is used for mapping and editing geographic data.

Arc Catalo gapplication is used for creating, previewing, documenting, organizing and managing geographic data.

Arc Tool box integrated application, is used for data editing, geoprocessing and spatial analysis.

Model Builder is used for designing and creating the study model.

\subsection{Data Collection}

The GIS-based decision support system developed in this study requires collecting spatial and non-spatial data. The spatial data such as the latitude and longitude of well-defined location points such as public schools, receiving hospitals and SRC centers in Jeddah were acquired with the help of GPS and Google Maps Application. The non-spatial data (attribute data) were collected in person.

\subsection{Data Processing and Analysis}

In this part of the study we determine the distance from the schools to the hospitals, which hospitals are nearest to the schools, and the direction from the hospitals to the schools. This will help care providers accurately determine estimated times of arrival and improve the efficiency of the service to reduce air ambulance response times.

\subsection{Distance Analysis}

Distance analysis is a form of spatial analysis that is commonly used to find shortest spatial distance between two points. ArcToolBox provides many spatial distance analysis tools one of the most common Distance Analysis tools is the Euclidean tools that are used to determine straight-line distance and direction or allocate resources based on cell proximity within a raster.

Three raster surfaces are created, using the straight-line Euclidean distance measurements.

These surfaces are then used to find out which of the hospitals is nearest to the school, how far the closest hospital is from the school, and in which direction the hospital is from the nearest school.

\subsubsection{Euclidean Distance Surface}

The Euclidean distance gives information according to Euclidean, or straight-line, distance [10]. It finds the shortest distance, a straight line, from each cell in a raster to the closest hospital (Figure 3). 


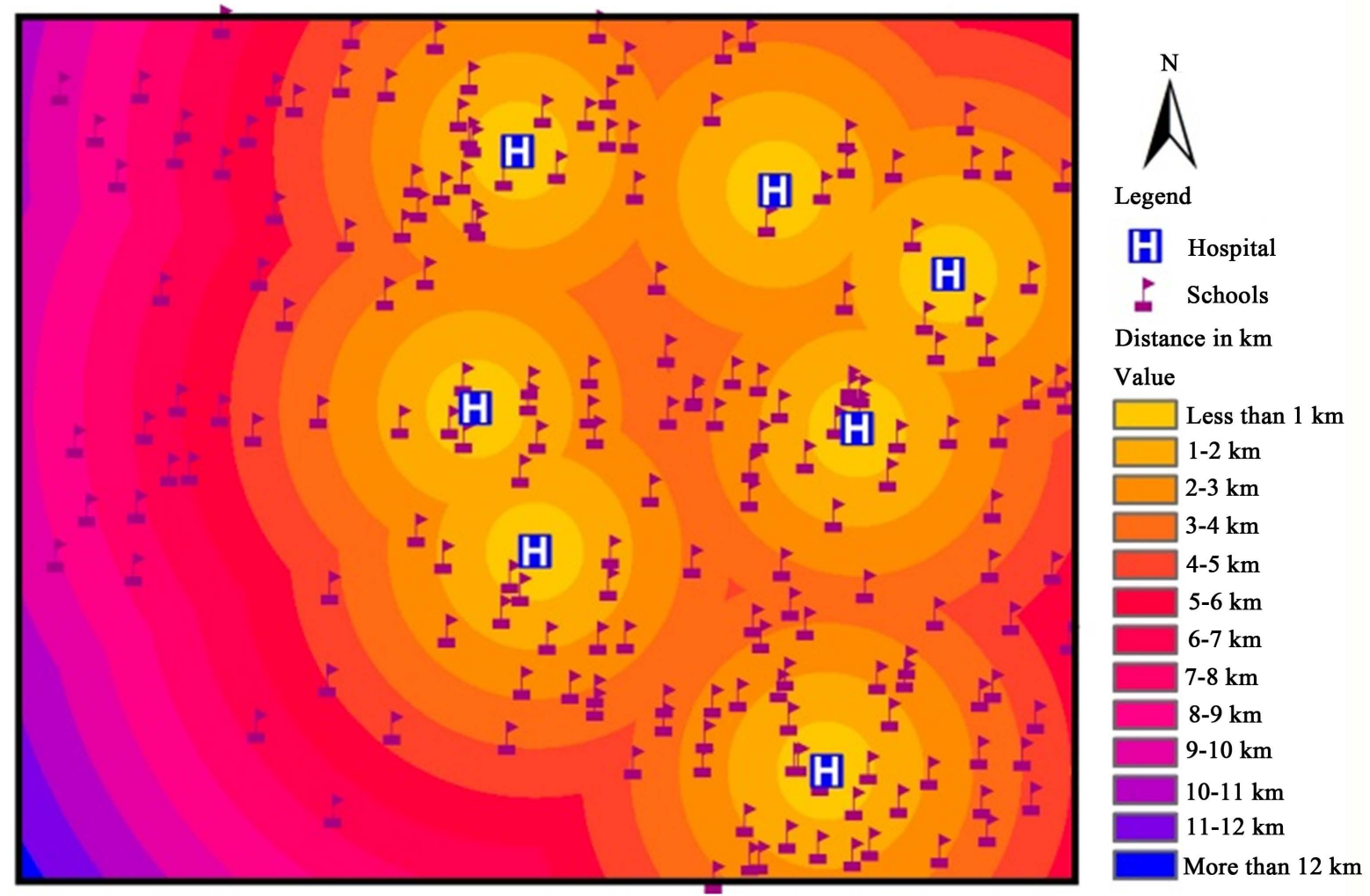

Figure 3. Euclidean distance surface.

\subsubsection{Euclidean Allocation Surface}

The Euclidean Allocation creates a raster surface where each cell is assigned to the nearest hospital based on the straight-line distance between them [10]. The surface is divided to zones of adjacent cells. These zones are linked to one hospital feature which is the nearest source (Figure 4).

\subsubsection{Euclidean Direction}

Euclidean direction raster assigns the direction of each cell in degrees to its nearest source along a straight line [10]. The cell values in a Euclidean direction surface point to the nearest hospital feature, indicating the direction, in compass directions (degrees azimuth), values range from 0 to 360 with north being 360 . Zero values indicate no direction. Cells that correspond to hospital locations have zero values in a straight-line direction surface or no direction (Figure 5).

\subsubsection{Reverse Direction Surface}

The Direction to hospital surface helps air ambulance transmitters direct pilots from a school location back to the nearest hospital. However, Transmitters must first direct the pilots from the hospital to the school. In this surface all the values in the Direction to hospital surface point in the opposite direction (180 degrees) (Figure 6).

Cell values for the reverse direction surface must be plus or minus 180 degrees from the Direction to hospital surface values and cannot have values over 360 degrees.

The CON tool in Map Algebra is used to create the reverse direction surface from the existing Direction to hospital surface. The Raster Calculator tool executes Map Algebra expressions using the python syntax [11].

The Map Algebra expression used for creating the reverse direction raster:

Con (("Direction to hospital" >0) \& ("Direction to hospital" $\leq 180$ ), "Direction to hospital" + 180, Con ("Direction to hospital" $>180$, "Direction to hospital" - 180.0))

IF the cell values in the Direction to hospital surface are greater than zero, but less than or equal to 180 , THEN add 180 to each cell value; otherwise, if cell values are greater than 180, subtract 180 from each cell value.If cell values do not match any of these criteria, give them a value of zero. 


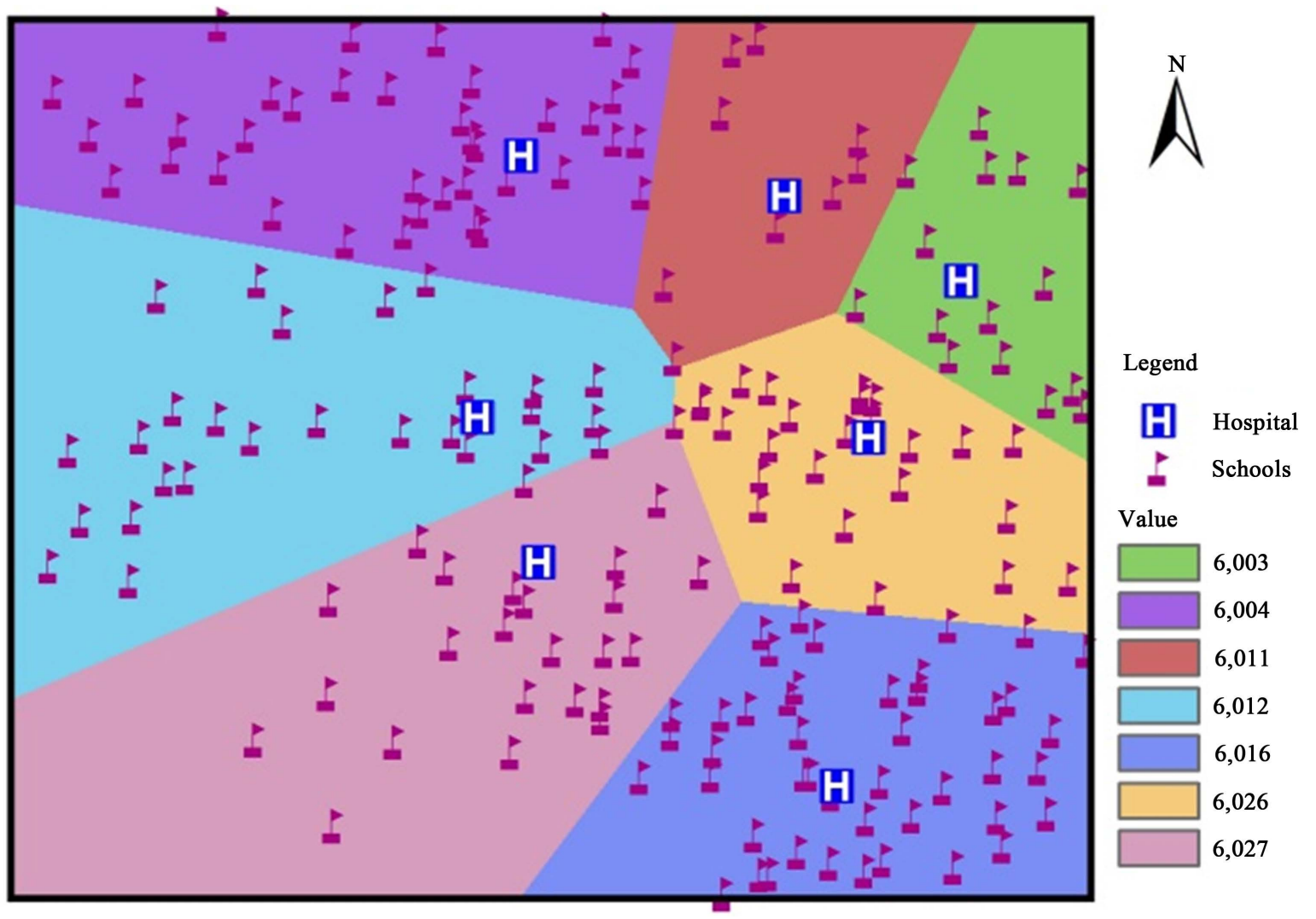

Figure 4. Euclidean allocation surface.
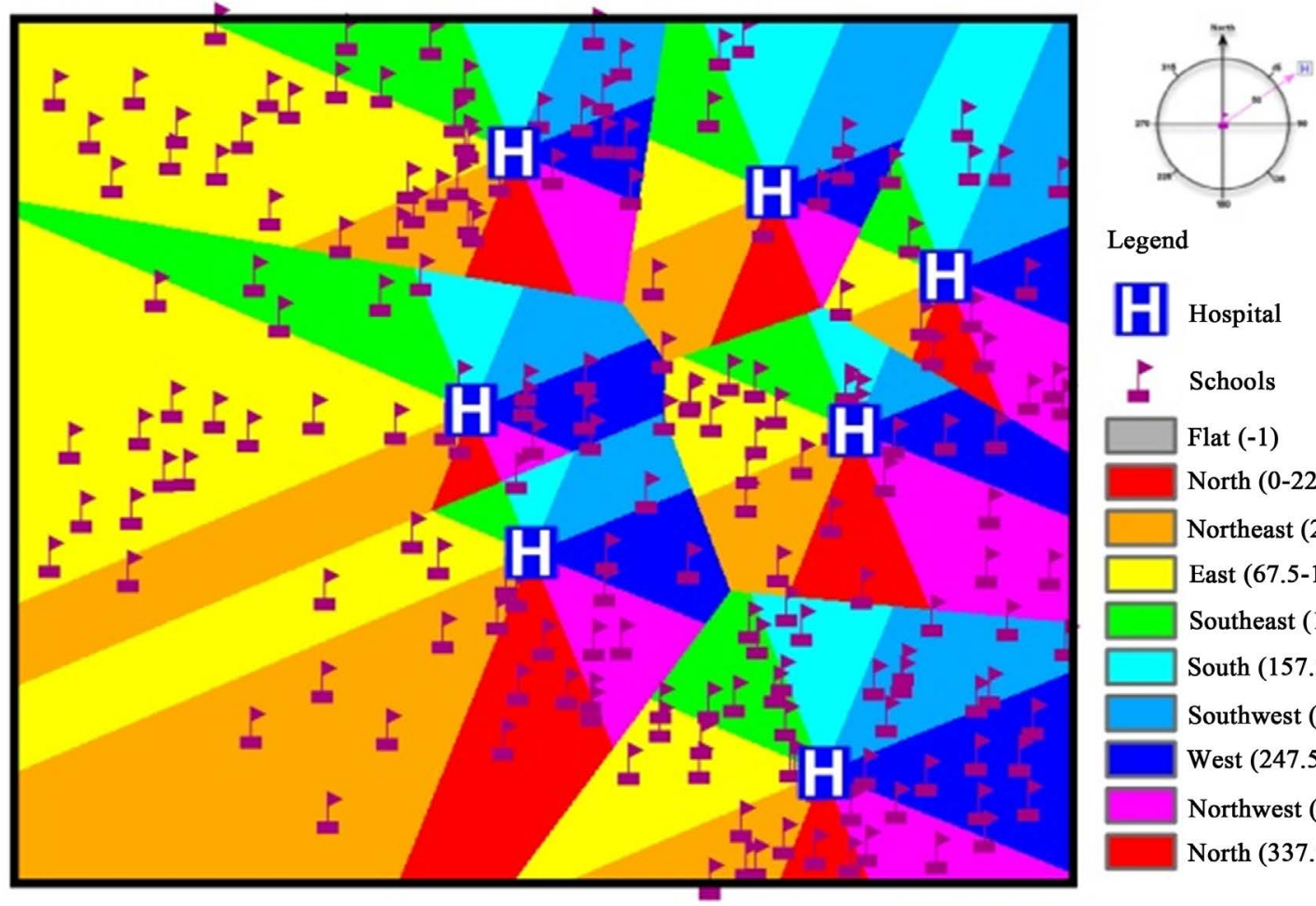

Legend

E. Hospital

Schools

Flat (-1)

North (0-22.5)

Northeast (22.5-67.5)

East (67.5-112.5)

Southeast (112.5-157.5)

South (157.5-202.5)

Southwest (202.5-247.5)

West (247.5-292.5)

Northwest (292.5-337.5)

North (337.5-360)

Figure 5. Euclidean direction surface. 


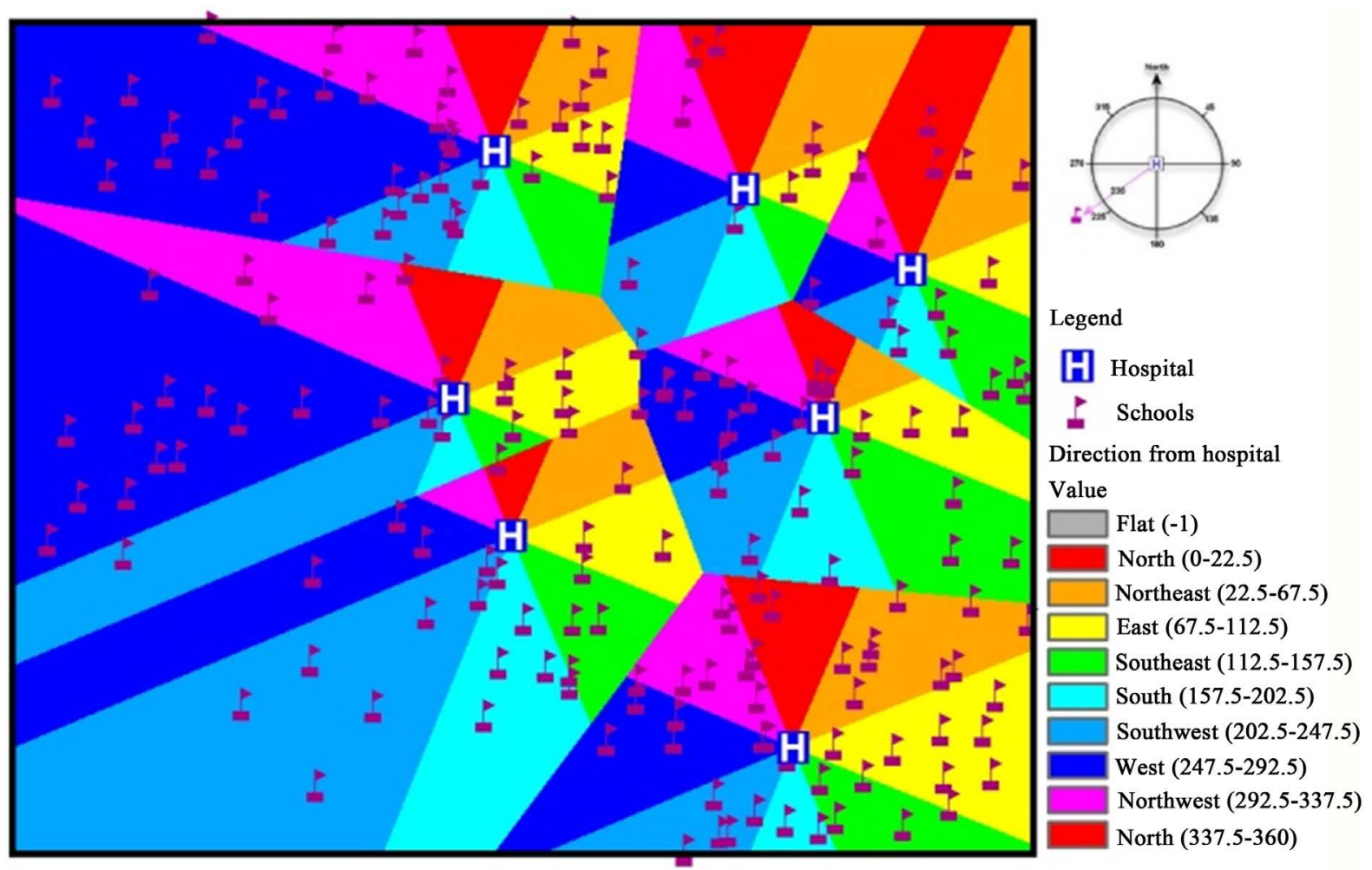

Figure 6. Euclidean reverse direction surface.

\subsection{Decision Support System}

The decision support system (DSS) for reducing air ambulance response time was executed by ArcInfo ArcGIS 10 software. "This software offers a possibility to design the steps of data processing by data flow diagram in the graphic editor ModelBuilder" [12].

The data flow diagram of the geoprocessing model could be automatically converted to Python a programing languageto easily add-on the programming scriptto other program codes.

The study used ModelBuilder to create the model to give a visual representation of the geoprocessing analysis operations, automate the geoprocessing workflow and to easily share it to other users.

The DSS implemented model consists of one input data (hospital vector data), four geoprocessing operations (spatial analysis tools and raster calculator tool), and four outputs of raster surfaces (Allocation, distance in $\mathrm{km}$, direction to hospital and direction from hospital) (Figure 7).

The spatial analysis tools used:

- Euclidean Allocation tool outputs three raster surfaces (Allocation, Direction and Distance calculated in map units which are feet).

- Divide tool converts distance unit from feet to $\mathrm{km}$ by dividing the raster by the constant value 3280.84 .

- Reclassify tool reclassifies the Distance in kilometers to hospital layer to allow dispatchers to visually assess the approximate distances to schools in easier-to-read zones. The dispatcher could look at the map and quickly determine that a particular school is approximately six kilometers the nearest hospital. The exact distance values will still be contained in the Distance in $\mathrm{km}$ to hospital surface.

The other used geoprocessing operation is the Raster Calculator tool which executes Map Algebra expressions using Python syntax to calculate the reversed direction.

\section{Conclusions and Recommendations}

GIS technology can be used to develop an efficient DSS to outline where air ambulance can reduce response times, by creating surfaces of Euclidean allocation, direction, and distance that can be used to improve initial response times for the civil defense air rescue and air ambulance services. 


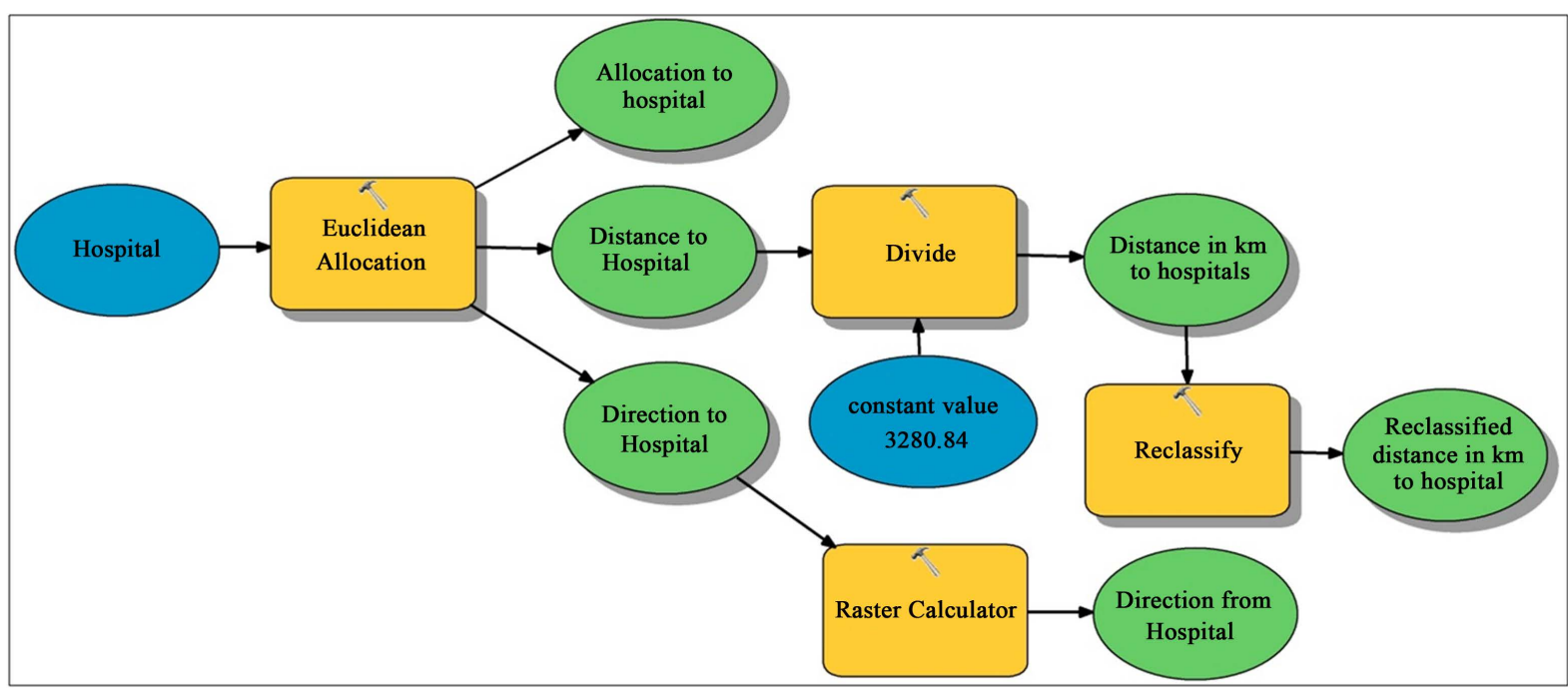

Figure 7. The implemented model.

Despite the fact that the developed DSS is a valuable tool for reducing response times, to increase its usefulness helicopter landing zones has to be considered. It is therefore suggested that more work be carried out to refine the model and to include remote sensing data like suitable landing zones near schools.

\section{References}

[1] Ong, M.E.H., et al. (2010) Reducing Ambulance Response Times Using Geospatial-Time Analysis of Ambulance Deployment. Academic Emergency Medicine, 17, 951-957. http://dx.doi.org/10.1111/j.1553-2712.2010.00860.x

[2] Harmon, J.E. and Anderson, S.J. (2003) The Design and Implementation of Geographic Information Systems. John Wiley \& Sons, Hobokon.

[3] Gunes, A.E. and Kovel, J.P. (2000) Using GIS in Emergency Management Operations. Journal of Urban Planning and Development, 126, 136-149. http://dx.doi.org/10.1061/(ASCE)0733-9488(2000)126:3(136)

[4] McGregor, J., et al. (2004) If All Ambulances Could Fly: Putting Provincial Standards of Emergency Care Access to the Test in Northern British Columbia. Canadian Journal of Rural Medicine: The Official Journal of the Society of Rural Physicians of Canada = Journal canadien de la medecine rurale: Le journal officiel de la Societe de medecine rurale du Canada, 10, 163-168.

[5] Panahi, S. and Delavar, M. (2009) Dynamic Shortest Path in Ambulance Routing Based on GIS. International Journal of Geoinformatics, 5, 13-19.

[6] Schuurman, N., et al. (2009) Modelling Optimal Location for Pre-Hospital Helicopter Emergency Medical Services. BMC Emergency Medicine, 9, 6. http://dx.doi.org/10.1186/1471-227X-9-6

[7] Lerner, E.B., et al. (1999) Use of a Geographic Information System to Determine Appropriate Means of Trauma Patient Transport. Academic Emergency Medicine, 6, 1127-1133. http://dx.doi.org/10.1111/j.1553-2712.1999.tb00115.x

[8] Al-Rashdi, M.R. (2011) Geographic Information System Saudi Red Crescent Authority in Jeddah: Analytical and Evaluative Study. Book of Conference.

[9] Ormsby, T., et al. (2001) Getting to Know ArcGIS Desktop. The Jste Teacher Education, 93.

[10] McCoy, J., et al. (2001) Using ArcGIS Spatial Analyst. Environmental Systems Research Institute. Inc., Redlands.

[11] ArcGIS, E. (2001) 8.3 of ArcMap, ArcCatalog, ArcToolbox and Spatial Analyst User's Guide. Redlands.

[12] Dobesova, Z. (2011) Programming Language Python for Data Processing. International Conference on Electrical and Control Engineering (ICECE), Yichang, 16-18 September 2011, 4866-4869. 